\title{
Rate Description of Fokker-Planck Processes with Time Dependent Parameters
}

\author{
Peter Talkner \\ Universität Augsburg, Institut für Physik, \\ Universitätsstrasse 1, D-86135 Augsburg, Germany \\ Jerzy Łuczka \\ Institute of Physics, University of Silesia, 40-007 Katowice, Poland
}

(Dated: September 4, 2018)

\begin{abstract}
The reduction of a continuous Markov process with multiple metastable states to a discrete rate process is investigated in the presence of slow time dependent parameters such as periodic external forces or slowly fluctuating barrier heights. A quantitative criterion is provided under which condition a kinetic description with time dependent frozen rates applies. Finally it is shown how the long time behavior of the underlying continuous process can be retrieved from the knowledge of the discrete process by means of an appropriate random decoration of the discrete states. As a particular example of the presented theory an over-damped bistable Brownian oscillator with periodic driving is discussed.
\end{abstract}

PACS numbers: 02.50.Ga, 82.20.Uv, 82.37.-j, 82.39.Rt 


\section{INTRODUCTION}

The separation of time scales is a frequently met dynamical feature of physical, chemical and biological processes. In practically all cases the parameters characterizing a particular system are only constant on a limited time scale. On longer time scales they may change their values because the surrounding environment slowly changes its state. We just note that even the most fundamental "constants" of nature like e.g. the fine structure constant possibly undergo a very slow change in time [1]. On the other hand, most natural processes also display very fast changes which usually will be disregarded in a phenomenological description. They only will show up in a microscopic description in which the effective degrees of freedom of the phenomenological model are represented as functions of microscopic degrees of freedom. The time evolution of these microscopic degrees of freedom typically takes place on much faster time scales than those of the macroscopic observables whereas, as already mentioned, global aspects of the environment may undergo temporal variations that are much slower than the dynamics of the considered macroscopic system.

It is this separation of time scales that allows one to describe a system in terms of a comparatively simple model that is closed in the sense that only a few macroscopic observables are subject to the dynamics. On the slow time scale the key technical notion here is that of a constraint equilibrium that is reached by the fast part of a system before the slow constituents change their values on the slower time scale. This constraint equilibrium then immediately adapts to the changes of the slow system.

This approach has been applied in many branches of physics and other sciences and is vital for various methods like adiabatic elimination, averaging, synergetics, subdynamics, chemical kinetics and hierarchical computational schemes [2]. In the present paper we consider Markovian processes the dynamics of which can be subdivided into a fast and a slow component. In particular, we have in mind the case where the slow motion is caused by transitions between metastable states [3]. The relaxation toward these states constitutes the fast part of the dynamics. Additionally, some of the system's parameters may undergo slow variations in time that may be caused by a slow drift, or fluctuations of the parameters, or by an external driving of the system. The dynamics of the considered system then has two slow components: The transitions and the driving. About their relation we do not make further assumptions: The slow intrinsic dynamics can be faster or slower than the external 
driving that must only be slow compared to the fast dynamics of the system. In Ref. [4] we have called this type of situation the semiadiabatic limit, in contrast to the adiabatic limit in which the driving is slow compared to the total intrinsic dynamics including that of the transitions. Clearly, the adiabatic limit is contained in the semiadiabatic limit as a special case.

Stochastic resonance is just one example for which the semiadiabatic limit is of relevance when the period of the signal is large [5, 6]. Ratchets, or Brownian motors, provide another class of systems which also operate in the semiadiabatic regime [7]. Atomic force microscopes and optical tweezers often act on time scales that are slow compared to the vibrational and other intrinsic time-scales of the molecules that are manipulated by them [8].

The paper is organized as follows. In Sect. II the reduction of a Fokker-Planck process with multiple mestastable states to a master equation is reviewed as it was developed in Refs. $[9,10]$. This method is generalized to processes with slowly time dependent parameters in Sect. III. The time rate of change of the probability now consists of two contributions. One is given by the rate as it would result if the parameters of the process were frozen and the other contribution mainly takes into account the changes of the geometry of the domains of attraction of the metastable states. This geometric contribution is proportional to the time rate of change of the parameters and therefore is negligible compared to the frozen rates if the time dependent parameters change sufficiently slowly. In contrast to previous investigations, [11] we find that the fastest time dependence of the parameters for which one may neglect the geometric contributions is not only determined by the deterministic time scales of the process but in general also depends on the strength of the noise. The way how the noise here enters depends on the particular process.

The present investigation is complementary to previous work that is restricted to periodic driving with an intermediate regime of external driving frequencies [12].

In Sect. IV we assume the validity of the master equation with frozen rates and determine a time dependent decoration of the discrete states such that the dynamics of the continuous variables of the underlying Fokker-Planck process are recovered on the long time scale. The archetypical example for stochastic resonance namely that of a periodically damped bistable Brownian oscillator is discussed in Sect. V and the validity of the McNamara-Wiesenfeld model of stochastic resonance [13] is discussed. The paper ends with a summary in Sect. VI. 


\section{MULTISTABLE SYSTEMS AT SMALL NOISE}

In this section we review the reduction of a Fokker-Planck equation describing a timehomogeneous process with multiple metastable states to a master equation that gives the dynamics on the time scales of the transitions between the metastable states. For this purpose we consider a dynamical system under the influence of weak random forces and assume that these forces can be modeled by Gaussian white noise. Then the stochastic dynamics is described by a Fokker-Planck equation [14]:

$$
\frac{\partial}{\partial t} \rho(\mathbf{x}, t)=L \rho(\mathbf{x}, t)
$$

where $L$ denotes the Fokker-Planck operator:

$$
L=-\sum_{i} \frac{\partial}{\partial x_{i}} K_{i}(\mathbf{x})+\sum_{i, j} \frac{\partial^{2}}{\partial x_{i} \partial x_{j}} D_{i j}(\mathbf{x})
$$

Here, $\mathbf{x}=\left(x_{1}, x_{2}, \ldots x_{n}\right)$ denotes a point with coordinates $x_{i}$ in the $n$-dimensional state space $\Sigma$; further, $\mathbf{K}(\mathbf{x})=\left(K_{i}(\mathbf{x})\right)$ is the drift vector and $\mathbf{D}(\mathbf{x})=\left(D_{i, j}(\mathbf{x})\right)$ the diffusion matrix resulting from the random forces. We further restrict ourselves to systems with a uniquely defined stationary probability density $\rho_{0}(\mathbf{x})$ with respect to which detailed balance holds. Consequently, the Fokker-Planck operator satisfies the relation [14]:

$$
L \hat{\rho}_{0}=\hat{\rho}_{0} \tilde{L}^{+}
$$

where $L^{+}$is the backward, or adjoint Fokker-Planck operator:

$$
L^{+}=K_{i}(\mathbf{x}) \frac{\partial}{\partial x_{i}}+D_{i, j}(\mathbf{x}) \frac{\partial^{2}}{\partial x_{i} \partial x_{j}} .
$$

The tilde denotes the operation of time reversal, i.e. $\tilde{x}_{i}=\epsilon_{i} x_{i}$ with parity $\epsilon_{i}= \pm 1$ and $\hat{\rho}_{0}$ is the multiplication operator with the probability density, i.e. $\hat{\rho}_{0} f(\mathbf{x})=\rho_{0}(\mathbf{x}) f(\mathbf{x})$ where $f(\mathbf{x})$ is an arbitrary state space function.

In the deterministic limit the diffusion matrix goes to zero and the drift vector approaches the deterministic vector field $\mathbf{K}^{(0)}(\mathbf{x})$ governing the deterministic motion of the system:

$$
\dot{\mathbf{x}}(t)=\mathbf{K}^{(0)}(\mathbf{x}(t))
$$

In the presence of weak random perturbations $\mathbf{K}(\mathbf{x})$ may differ from $\mathbf{K}^{0}(\mathbf{x})$ by small noise induced contributions. 


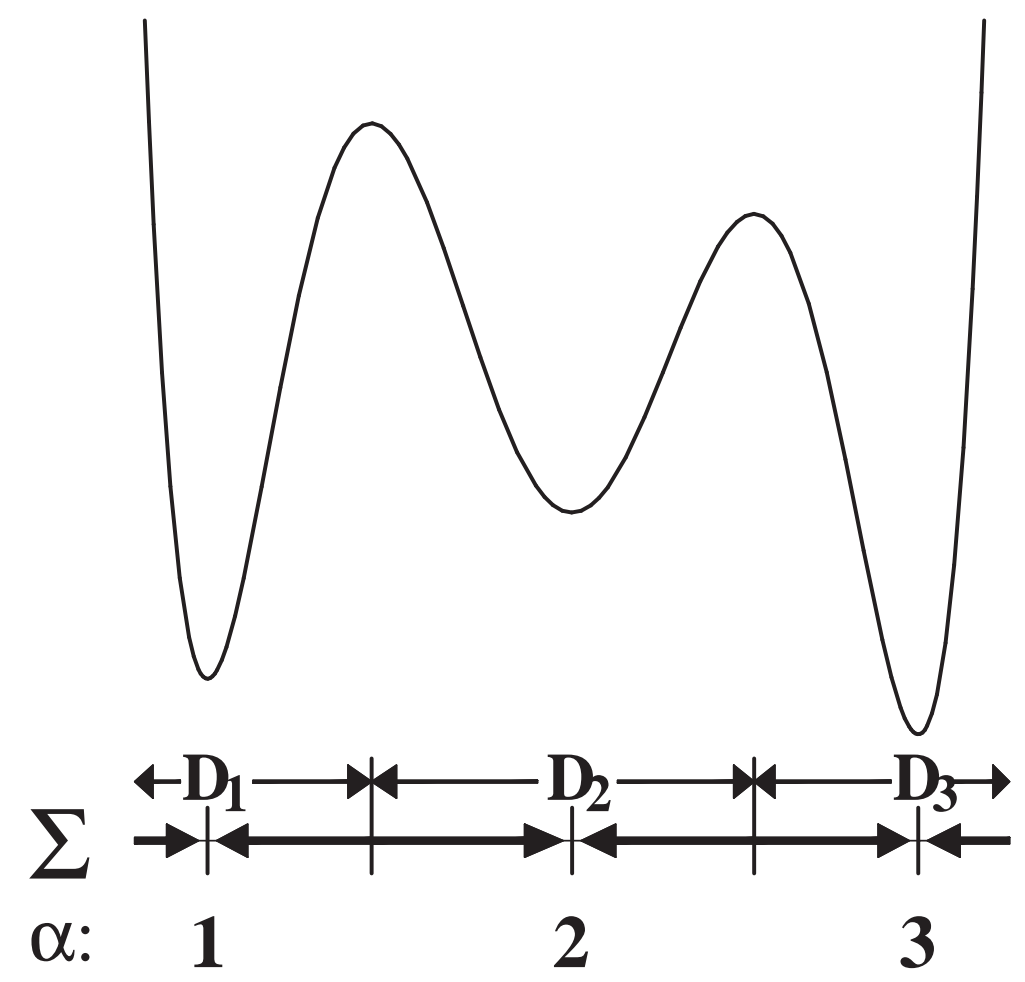

FIG. 1: The overdamped deterministic motion $\dot{x}(t)=-V^{\prime}(x(t))$ in the above shown potential induces a partitioning of the state space $\Sigma=\mathbb{R}_{1}$ into the domains of attraction $\mathcal{D}_{\alpha}$ of the three attractors $\alpha=1,2,3$ at the potential minima. Here the locations of the local potential maxima coincide with the boundaries between the domains of attraction.

We here are interested in cases where the deterministic system (5) has a number $m$ of coexisting attractors labeled by $\alpha$. To each attractor $\alpha$ there belongs a domain of attraction $\mathcal{D}_{\alpha}$. The domains are disjoint and partition the total available state space:

$$
\begin{aligned}
\mathcal{D}_{\alpha} \bigcap \mathcal{D}_{\alpha^{\prime}} & =\emptyset \quad \text { for } \alpha \neq \alpha^{\prime} \\
\bigcup_{\alpha=1}^{m} \mathcal{D}_{\alpha} & =\Sigma
\end{aligned}
$$

For weak noise the stationary probability density is almost zero everywhere except close to the attractors. If the noise vanishes the stationary density shrinks to Dirac $\delta$-functions sitting at the attractors of the deterministic system. Because no transitions between the different attractors may occur in the noiseless case, each of the delta functions is a stationary solution of the Fokker-Planck equation $\partial / \partial x_{i} K_{i}^{0}(\mathbf{x}) \rho_{0}(\mathbf{x})=0$ belonging to a $m$-fold degenerate eigenvalue 0.

The degeneracy also follows from the backward equation which is governed by the adjoint 
Fokker-Planck operator $L^{+}$defined in eq. (4). In the deterministic case the diffusion matrix vanishes, so the second derivatives disappear and the stationary solutions of the backward operator are constant along the trajectories of the deterministic system. Because within a domain of attraction all trajectories approach the same attractor the stationary solutions of the noiseless backward equation are constant on the domain of attraction $\mathcal{D}_{\alpha}$. One then can choose the characteristic functions $\chi_{\alpha}^{(0)}(\mathbf{x})$ of the domains of attraction as $m$ independent solutions of the stationary deterministic backward equation $K_{i}^{(0)}(\mathbf{x}) \partial \chi^{(0)}(\mathbf{x}) / \partial x_{i}=0$ :

$$
\chi_{\alpha}^{(0)}(\mathbf{x})= \begin{cases}1 & \text { for } \mathbf{x} \in \mathcal{D}_{\alpha} \\ 0 & \text { else }\end{cases}
$$

In the presence of weak noise these functions can be modified slightly such that they still solve the stationary backward equation up to corrections that are exponentially small in the noise strength: The step-like discontinuities then are smeared out on layers located at the boundaries of the domains of attraction $\mathcal{D}_{\alpha}$. The functions can be constructed by means of the method of matched asymptotic expansions [15], see also Refs. [9, 10]. Outside the boundary layers no modification is required and hence they retain their property of localizing the different domains of attraction. In the following, these localizing functions will be denoted by $\chi_{\alpha}(\mathbf{x})$. We summarize their main properties: They solve $L^{+} \chi_{\alpha}(\mathbf{x})=0$ up to exponentially small corrections in the noise strength, have the values one within and zero outside $\mathcal{D}_{\alpha}$ and interpolate between these values smoothly on a thin boundary layer $[16,17]$. As solutions of these particular boundary value problems, the functions $\chi_{\alpha}(\mathbf{x})$ are the splitting probabilities that give the relative frequencies with which a trajectory starting at $\mathbf{x}$ first reaches the attractor $\alpha$ before it visits any other attractor [18].

The splitting probabilities in general are not eigenfunctions of the backward operator. However, they span the subspace of eigenfunctions that emerges from the eigenspace of the $m$-fold degenerate eigenvalue 0 of the deterministic dynamics if weak noise perturbes the system and lifts the degeneracy. The resulting finite eigenvalues are still small compared to all other finite eigenvalues. Hence, this subspace describes the slow dynamics of the system at weak noise. In particular, it contains the constant function which is the exact eigenfunction of the backward equation belonging to the eigenvalue 0 . At weak noise this eigenfunction can be represented as the sum over the characteristic functions $\chi_{\alpha}(\mathbf{x})$ :

$$
\sum_{\alpha} \chi_{\alpha}(\mathbf{x})=1
$$


Because of detailed balance, the slow subspace of the forward operator is spanned by the basis set $\left\{\tilde{\chi}_{\alpha}(\mathbf{x}) \rho_{0}(\mathbf{x})\right\}$, where $\tilde{\chi}_{\alpha}(\mathbf{x})=\chi_{\alpha}(\tilde{\mathbf{x}})$ denotes the image of $\chi_{a}(\mathbf{x})$ under time reversal. Hence, the probability density describing the slow dynamics can be represented as:

$$
\rho(\mathbf{x}, t)=\sum_{\alpha=1}^{m} c_{\alpha}(t) \tilde{\chi}_{\alpha}(\mathbf{x}) \rho_{0}(\mathbf{x})
$$

As already noted the characteristic functions $\chi_{\alpha}(\mathbf{x})$ and, therefore also their time reversed partners $\tilde{\chi}_{\alpha}(\mathbf{x})$, are well localized functions as long as the noise does not become too large. As a consequence, one may use the functions $\chi_{\alpha}(\mathbf{x})$ in order to determine the probability $p_{\alpha}(t)$ that the system resides in the domain of attraction of the attractor $\alpha[10]$ :

$$
p_{\alpha}(t)=\left(\chi_{\alpha}, \rho(t)\right)
$$

where the scalar product is defined as the integral of the product of its arguments over the state space:

$$
(f, g)=\int_{\Sigma} d^{n} \mathbf{x} f(\mathbf{x}) g(\mathbf{x}) .
$$

If we assume that the probability density $\rho(t)$ is of the form (10) we obtain

$$
p_{\alpha}(t)=c_{\alpha}(t) n_{\alpha}
$$

where we have made use of the localization of the characteristic functions at weak noise:

$$
\left(\chi_{\alpha}, \tilde{\chi}_{\alpha^{\prime}} \rho_{0}\right)=\delta_{\alpha, \alpha^{\prime}} n_{\alpha}
$$

and have introduced the function $n_{\alpha}=\int d x \chi_{\alpha}(x) \tilde{\chi}_{\alpha}(x) \rho_{0}(x) \approx \int d x \chi_{\alpha}(x) \rho_{0}(x)$ giving the population of the metastable state $\alpha$ in the equilibrium distribution $\rho_{0}(x)$.

The time evolution of the probabilities $p_{\alpha}(t)$ now follows from the projection of the Fokker-Planck equation (1) onto the slow subspace, i.e. we assume the probability density to take the form (10) and determine the scalar product with $\chi_{\alpha}(\mathbf{x})$ on both sides of the Fokker-Planck equation. This yields

$$
\frac{d}{d t} p_{\alpha}(t)=\sum_{\alpha^{\prime}} \frac{\left(\chi_{a}, L \tilde{\chi}_{\alpha^{\prime}} \rho_{0}\right)}{n_{\alpha^{\prime}}} p_{\alpha^{\prime}}(t)
$$

Under the sum on the right hand side we expressed the coefficients $c_{\alpha^{\prime}}(t)$ by means of eq. (13) in terms of the probabilities $p_{\alpha^{\prime}}(t)$ leading to the equilibrium weights $n_{\alpha^{\prime}}$ in the denominator of the respective coefficient. For $\alpha \neq \alpha^{\prime}$ the coefficient $\left(\chi_{\alpha}, L \tilde{\chi}_{\alpha^{\prime}} \rho_{0}\right) / n_{\alpha^{\prime}}$ coincides with the 
Rayleigh-quotient expressions for the rate from $\alpha^{\prime}$ to $\alpha$ [17]. The integral is dominated by a neighborhood of the saddle point that connects the domains of attraction $\mathcal{D}_{\alpha^{\prime}}$ and $\mathcal{D}_{\alpha}$ [19]. Within this neighborhood, for small noise, the localizing function $\chi_{\alpha}(\mathbf{x})$ can be approximated by the characteristic function of $\mathcal{D}_{\alpha}, \chi_{\alpha}^{0}(x)[20]$. The resulting coefficient then coincides with the better known flux-over-population expression for the respective rate [3]. In particular, one can show that for sufficiently weak noise the expression $\left(\chi_{\alpha}, L \tilde{\chi}_{\alpha^{\prime}} \rho_{0}\right) / n_{\alpha^{\prime}}$ is positive for $\alpha \neq \alpha^{\prime}$. From eq. (9) it follows that the sum of the coefficients over $\alpha$ vanishes. Hence, eq. (15) has the proper form of a master equation:

$$
\frac{d}{d t} p_{\alpha}(t)=\sum_{\alpha^{\prime} \neq \alpha} r_{\alpha, \alpha^{\prime}} p_{\alpha^{\prime}}(t)-\sum_{\alpha^{\prime} \neq \alpha} r_{\alpha^{\prime}, \alpha} p_{\alpha}(t)
$$

where

$$
r_{\alpha, \alpha^{\prime}}=\frac{\left(\chi_{\alpha}, L \tilde{\chi}_{\alpha^{\prime}} \rho_{0}\right)}{n_{\alpha^{\prime}}}
$$

is the transition probability per unit time from $\alpha^{\prime}$ to $\alpha$.

The regime of validity of this approximation, of course, depends on the particular system and the degree of accuracy one would like to achieve. By its very nature this master equation is only appropriate for the long time dynamics of the Fokker-Planck equation and therefore requires a clear separation of time scales dividing the process into fast relaxations within the domains of attraction and slow transitions between the attractors. We note that various definitions of transition rates exist which are different from a physical point of view but which still yield the same result if a master equation provides a correct description of the long time dynamics. It turns out that the actual differences between these rate expressions are exponentially small in the noise strength, i.e. of the form $\exp \left\{-\Delta V / k_{B} T\right\}$ where $\Delta V$ is a relevant barrier hight and $k_{B} T$ the noise strength. So if we are ready to accept an error of, say $1 \%$, all barriers in the system must be higher than approximately $4.5 k_{B} T$.

\section{SLOW DRIVING}

After these preparatory considerations we turn to our main topic and consider a FokkerPlanck process of the kind of the previous section with the only difference that we now allow for slow changes of the parameters of the system. These could result from a slow drift of the environmental parameters of the system like e.g. the temperature, a slow increase of an external field, or a slow periodic force driving the system, to name but a few realizations. 
We emphasize that the change of the parameters must be slow compared to the fast relaxation within each domain of attraction but no assumption is made how it compares to the slow intrinsic dynamics of the system describing the transitions between different domains of attraction. We assume that in the presence of these time dependent parameters one still can identify domains of attraction which do not merge or split as time changes but retain their identity. In other words, we restrict ourselves to changes of the parameters that do not lead to topological changes of the system.

The resulting process is still Markovian and is governed by a Fokker-Planck equation:

$$
\frac{\partial}{\partial t} \rho(t)=L(t) \rho(t)
$$

where the Fokker-Planck operator $L(t)$ is of the form given in eq. (2) with time dependent drift $\mathbf{K}(\mathbf{x}, t)$ and possibly also a time dependent diffusion matrix $\mathbf{D}(\mathbf{x}, t)$. In particular, we assume that all parameter values which the system experiences in the course of time correspond to equilibrium systems, i.e. that for any frozen parameter value the system will reach an equilibrium distribution relative to which the system obeys detailed balance:

$$
L(t) \hat{\rho}_{0}(t)=\hat{\rho}_{0}(t) \tilde{L}^{+}(t)
$$

where the tilde denotes time reversal and $\hat{\rho}_{0}(t)$ is the multiplication operator with the equilibrium distribution $\rho_{0}(\mathbf{x}, t)$ that would be attained if the parameter values were frozen at the values that they assume at time $t$ :

$$
L(t) \rho_{0}(\mathbf{x}, t)=0 .
$$

For vanishing noise the system will move toward the nearest attractor as defined by the momentary values of the parameters. During this relaxation time, the parameters of the system are supposed to change by such a small amount that they can be considered as kept constant. Only when the system has reached the attractor and stays there for a much longer time, the change of parameters will become noticeable and the system will follow the slow motion of the attractor as it results from the changing parameters. This means that the time rate of change of the deterministic drift is characterized by a small parameter $\epsilon$ :

$$
\frac{|\dot{\mathbf{K}}(\mathbf{x}, t)|}{|\mathbf{K}(\mathbf{x}, t)|} \tau_{r}=\mathcal{O}(\epsilon)
$$


where $\tau_{r}$ is a typical relaxation time of the deterministic motion. Under this very condition many of the properties of the time homogeneous process carry over to the case with time dependent parameters.

To each attractor $\alpha$ then there belongs a domain of attraction $\mathcal{D}_{\alpha}(t)$ together with its characteristic function $\chi_{\alpha}^{(0)}(\mathbf{x}, t)$ which is 1 on $\mathcal{D}_{\alpha}(t)$ and 0 elsewhere in $\Sigma$. The domains of attractions also slowly depend on time and so do the characteristic functions. If the system is weakly perturbed by noise the characteristic functions will change into smooth functions $\chi_{\alpha}(\mathbf{x}, t)$ that coincide with the noiseless functions everywhere except in a thin boundary layer at $\partial \mathcal{D}_{\alpha}(t)$, where a steep but smooth transition from 1 to 0 takes place. As in the time independent case, they are solutions of the homogeneous backward equation that approach 1 in the interior of $\mathcal{D}_{\alpha}(t)$ and vanish on all other attractors $\alpha^{\prime} \neq \alpha$. Further one also can represent the slow dynamics of the probability density in terms of linear combinations of $\tilde{\chi}_{\alpha}(\mathbf{x}, t) \rho_{0}(\mathbf{x}, t)$ :

$$
\rho(\mathbf{x}, t)=\sum_{\alpha=1}^{m} c_{\alpha}(t) \tilde{\chi}_{\alpha}(\mathbf{x}, t) \rho_{0}(\mathbf{x}, t)
$$

The probabilities to find the system in the state $\alpha$ is analogously defined as in the case with constant parameters:

$$
p_{\alpha}(t)=\left(\chi_{\alpha}(t), \rho(t)\right)
$$

The time rate of change of $p_{\alpha}(t)$ consequently has two contributions, resulting from the derivative of $\chi_{\alpha}(\mathbf{x}, t)$ and of $\rho_{0}(\mathbf{x}, t)$ :

$$
\frac{d}{d t} p_{\alpha}(t)=\left(\frac{\partial}{\partial} \chi_{\alpha}(t), \rho(t)\right)+\left(\chi_{\alpha}(t), \frac{\partial}{\partial t} \rho(t)\right) .
$$

The latter probabilistic contribution can be expressed in terms of the Fokker-Planck equation yielding

$$
\left(\chi_{\alpha}, \frac{\partial}{\partial t} \rho(t)\right)=\sum_{\alpha^{\prime}} r_{\alpha, \alpha^{\prime}}(t) p_{\alpha}(t)
$$

where we used eq. (22) and introduced the time dependent transition rates $r_{\alpha, \alpha^{\prime}}(t)$ in an analogous way as in the time homogeneous case, see eq. (17):

$$
r_{\alpha, \alpha^{\prime}}(t)=\frac{\left(\chi_{\alpha}(t), L(t) \tilde{\chi}_{\alpha^{\prime}}(t) \rho_{0}(t)\right)}{n_{\alpha^{\prime}}(t)} .
$$

It determines the rate of change of the probability $p_{\alpha}(t)$ caused by transitions from $\alpha^{\prime}$ to $\alpha$. Here $n_{\alpha^{\prime}}(t)$ is the population of the metastable state $\alpha^{\prime}$ in the frozen equilibrium distribution 
$\rho_{0}(\mathbf{x}, t)$. It is given by:

$$
n_{\alpha}(t)=\left(\chi_{\alpha}(t), \tilde{\chi}_{\alpha}(t) \rho_{0}(t)\right) \approx\left(\chi_{\alpha}(t), \rho_{0}(t)\right)
$$

where the term $\tilde{\chi}_{\alpha}(\mathbf{x}, t)$ is neglected in the second equation. This approximation holds up to exponentially small terms in the noise strength.

The other contribution, $\left(\partial \chi_{\alpha}(t) / \partial t, \rho(t)\right)$, describes the change of the probability caused by the geometric change of the domain $\mathcal{D}_{\alpha}(t)$. It also is linear in the probabilities $p_{\alpha^{\prime}}(t)$ and can be written as

$$
\left(\frac{\partial}{\partial t} \chi_{\alpha}(t), \rho(t)\right)=\sum_{\alpha^{\prime}} g_{\alpha, \alpha^{\prime}}(t) p_{\alpha^{\prime}}(t)
$$

where

$$
g_{\alpha, \alpha^{\prime}}(t)=\frac{\left(\frac{\partial}{\partial t} \chi_{\alpha}(t), \tilde{\chi}_{\alpha^{\prime}}(t) \rho_{0}(t)\right)}{n_{\alpha^{\prime}}(t)}
$$

The coefficients $g_{\alpha, \alpha^{\prime}}(t)$ are proportional to the smallness parameter $\epsilon$ because they contain the time derivative of $\chi_{\alpha}(x, t)$. The proportionality factor roughly is of the same order of magnitude as the transition rate $r_{\alpha, \alpha^{\prime}}(t)$ :

$$
\frac{g_{\alpha, \alpha^{\prime}}(t)}{r_{\alpha, \alpha^{\prime}}(t)}=\mathcal{O}(\epsilon)
$$

The dependence of this ratio on the noise strength is discussed for a particular example in section V. For a sufficiently slow parameter change, i.e a small value of $\epsilon$, the geometric contribution to the time rate of change of the probability $p_{\alpha}(t)$ can be neglected and a master equation results that is completely determined by the instantaneous rates $r_{\alpha, \alpha^{\prime}}(t)$ :

$$
\frac{d}{d t} p_{\alpha}(t)=\sum_{\alpha^{\prime} \neq \alpha} r_{\alpha, \alpha^{\prime}}(t) p_{\alpha^{\prime}}(t)-\sum_{\alpha^{\prime} \neq \alpha} r_{\alpha^{\prime}, \alpha}(t) p_{\alpha}(t)
$$

where we used that the time dependent rates $r_{\alpha, \alpha^{\prime}}(t)(26)$ have the same general form and therefore the same formal properties as the time independent ones (17): Those for $\alpha \neq \alpha^{\prime}$ are positive and the sum over the first index vanishes: $\sum_{\alpha} r_{\alpha, \alpha^{\prime}}(t)=0$. This master equation with time dependent rates is the central result of the present paper. For faster driving the geometric contributions of the rates have to be taken into account. However, they may become negative, and therefore formally negative rates may result if the driving is too fast. This indicates that then the instananeous eigenfunctions of the Fokker Planck equation no longer provide a good basis. 


\section{DECORATING THE META-STABLE STATES}

Often the knowledge of the probabilities $p_{\alpha}(t)$ to find the system at time $t$ in the metastable state $\alpha$ is not sufficient. For example one may be interested in the average position $\mathbf{x}$ of the system or its single- or multi-time statistical properties. We here show how the time dependence of these quantities on the slow time scales of the transitions and the external driving can be retrieved from exactly the same information that is necessary to determine the transition rates from the Fokker-Planck equation. For that purpose we first consider the average of an arbitrary function $f(\mathbf{x})$ of the position:

$$
\begin{aligned}
\langle f(t)\rangle & =\int d^{n} \mathbf{x} f(\mathbf{x}) \rho(\mathbf{x}, t) \\
& =\sum_{\alpha}\langle f(t) \mid \alpha\rangle p_{\alpha}(t)
\end{aligned}
$$

where $\langle f(t) \mid \alpha\rangle$ denotes the expectation value of $f(x)$ under the condition that the system resides at $t$ in the domain of attraction $\mathcal{D}_{\alpha}(t)$. Using the long-time behavior of the probability density $\rho(t)$ as given by eq. (22) one finds:

$$
\langle f(t) \mid \alpha\rangle=\int d^{n} \mathbf{x} f(\mathbf{x}) \rho(\mathbf{x}, t \mid \alpha)
$$

where the conditional probability $\rho(\mathbf{x}, t \mid \alpha)$ to find the system in the continuous state $\mathbf{x} \in$ $\mathcal{D}_{\alpha}(t)$ is given by

$$
\rho(\mathbf{x}, t \mid \alpha)=\frac{\tilde{\chi}_{\alpha}(\mathbf{x}, t)}{n_{\alpha}(t)} \rho_{0}(\mathbf{x}, t)
$$

Similarly, one finds for the correlation of two functions $f(\mathbf{x})$ and $g(\mathbf{x})$ at times $t$ and $t+\tau$ being separated by a positive time $\tau$ that is long compared to the fast time scale of relaxations within the domains of attraction the following expression:

$$
\langle f(t+\tau) g(t)\rangle=\sum_{\alpha, \alpha^{\prime}}\langle f(t+\tau) \mid \alpha\rangle\left\langle g(t) \mid \alpha^{\prime}\right\rangle p\left(\alpha, t+\tau \mid \alpha^{\prime}, t\right) p_{\alpha^{\prime}}(t) .
$$

Here $p\left(\alpha, t+\tau \mid \alpha^{\prime}, t\right)$ is the conditional probability to find the system in the metastable state $\alpha$ at time $t+\tau$ provided it was in the state $\alpha^{\prime}$ at the earlier time time $t$. This conditional probability is the solution of the master equation (31) subject to the initial conditions $p\left(\alpha, t \mid \alpha^{\prime}, t\right)=\delta_{\alpha, \alpha^{\prime}}$. Eq. (35) holds for arbitrary functions $f(\mathbf{x})$ and $g(\mathbf{x})$. Therefore the conditional probability of finding the system at time $t+\tau$ at the continuous state $x$ if it was at the earlier time $t$ at $y$ takes the form

$$
\rho(\mathbf{x}, t+\tau \mid \mathbf{y}, t)=\sum_{\alpha, \alpha^{\prime}} \rho(\mathbf{x}, t+\tau \mid \alpha) \chi_{\alpha^{\prime}}(\mathbf{y}, t) p\left(\alpha, t+\tau \mid \alpha^{\prime}, t\right)
$$


For an independent derivation of this result see the Appendix A.

Yet another analogous way exists to characterize the continuous process on the long time scale: One takes the discrete process $z(t)$ assuming the values $\alpha=1 \ldots m$ according to the master equation (31) and decorates the states with a random point in the state space, $\mathbf{X}(z(t), t)$, depending on time and the particular state $\alpha=z(t)$ that is realized at the time $t$. If the probability density of the decoration $\mathbf{X}(\alpha, t)$ is chosen as the conditional probability density $\rho(\mathbf{x}, t \mid \alpha)$ given in eq. (34) the mean values and the correlation functions of the process $\mathbf{X}(\mathbf{x}, t)$ coincide with the expressions (32) and (35), respectively, and accordingly are characterized by the conditional probability (36). The decorated process is Markovian and has the same single and two time probability density as the continuous process $\mathbf{x}(t)$ on the long time scale and hence coincides there with it:

$$
\mathbf{x}(t)=\mathbf{X}(z(t), t)
$$

\section{BISTABLE OVERDAMPED OSCILLATOR}

We here consider the example of an overdamped bistable Brownian oscillator that is periodically driven at a frequency $\Omega$ which is slow compared to the typical relaxation rates. Its dynamics is given by the Smoluchowski operator

$$
L_{S}(t)=\frac{\partial}{\partial x} \frac{\partial V(x, t)}{\partial x}+\beta^{-1} \frac{\partial^{2}}{\partial x^{2}}
$$

where $\beta^{-1}$ is the noise strength which is proportional to the temperature $\theta$ of the fluid surrounding the oscillator, and $V(x, t)$ denotes the time-dependent potential:

$$
V(x, t)=\frac{1}{4} x^{4}-\frac{1}{2} x^{2}-A x \sin (\Omega t) .
$$

Here $A$ denotes the strength of the periodic force. Throughout dimensionless units are used. It is supposed to stay within the limits $|A|<2 /(3 \sqrt{3)}$. Actually it must keep some finite distance from these limits in order that the following asymptotic theory applies. Under this condition on the strength of the external force, the potential has three stationary points that are solutions of the algebraic equation:

$$
x^{3}-x=A \sin (\Omega t)
$$

As time varies they form three branches: $x_{1}(t)$ and $x_{2}(t)$ are those tracing the two local minima that at $t=0$ assume the values $x_{1}(0)=-1$ and $x_{2}(0)=1$, respectively, and $x_{b}(t)$ 
gives the location of the local potential maximum between the minima. At $t=0$ it is located at $x_{b}(0)=0$. The corresponding extreme values of the potential $V(x, t)$ are denoted by:

$$
\begin{aligned}
& V_{\alpha}(t)=V\left(x_{\alpha}(t), t\right) \quad \text { for } \alpha=1,2 \\
& V_{b}(t)=V\left(x_{b}(t), t\right)
\end{aligned}
$$

The instantaneous well and barrier frequencies are defined accordingly as:

$$
\begin{aligned}
& \omega_{\alpha}(t)=\sqrt{\frac{\partial^{2} V\left(x_{\alpha}(t), t\right)}{\partial x}}=\sqrt{3 x_{\alpha}^{2}(t)-1} \quad \text { for } \alpha=1,2 \\
& \omega_{b}(t)=\sqrt{\frac{-\partial^{2} V\left(x_{b}(t), t\right)}{\partial x}}=\sqrt{1-3 x_{b}^{2}(t)}
\end{aligned}
$$

For later use we give the time derivatives of the barrier position and the barrier frequencies. For the position one finds from eq. (40):

$$
\dot{x}_{b}(t)=-\frac{\Omega A}{\omega_{b}(t)^{2}} \cos (\Omega t)
$$

and for the frequency it follows:

$$
\dot{\omega}_{b}(t)=-3 \frac{x_{b}(t)}{\omega_{b}(t)} \dot{x}_{b}(t)
$$

The instantaneous stationary solution of the Smoluchowski equation $L_{S}(t) \rho_{0}(x, t)=0$ is given by the Boltzmann distribution:

$$
\rho_{0}(x, t)=Z^{-1}(t) e^{-\beta V(x, t)}
$$

where for small noise the partition function is given by

$$
Z(t)=\sqrt{\frac{2 \pi}{\beta}}\left\{\frac{\exp \left\{-\beta V_{1}(t)\right\}}{\omega_{1}}+\frac{\exp \left\{-\beta V_{2}(t)\right\}}{\omega_{2}}\right\}
$$

Here algebraic correction terms of the order $\mathcal{O}\left(1 /\left(\beta \Delta V_{\alpha}(t)\right)\right)$ were neglected. The domains of attraction of the instantaneous locally stable states $x_{\alpha}(t), \alpha=1,2$, extend from minus infinity to the instantaneous barrier and from there to infinity: $\mathcal{D}_{1}(t)=\left(-\infty, x_{b}(t)\right)$, and $\mathcal{D}_{2}(t)=\left(x_{b}(t), \infty\right)$.

The corresponding localizing functions $\chi_{\alpha}(x, t)$ are the solutions of the backward equation

$$
\left\{\omega_{b}(t)^{2}\left(x-x_{b}(t)\right) \frac{\partial}{\partial x}+\beta^{-1} \frac{\partial^{2}}{\partial x^{2}}\right\} \chi_{\alpha}(x, t)=0
$$


with boundary conditions:

$$
\begin{aligned}
& \chi_{1}(x, t)= \begin{cases}1 & \text { for } x \rightarrow-\infty \\
0 & \text { for } x \rightarrow \infty\end{cases} \\
& \chi_{2}(x, t)= \begin{cases}0 & \text { for } x \rightarrow-\infty \\
1 & \text { for } x \rightarrow \infty\end{cases}
\end{aligned}
$$

The solutions readily are found:

$$
\begin{aligned}
& \chi_{1}(x, t)=\frac{1}{2} \operatorname{erfc}\left(\omega_{b}(t) \sqrt{\beta / 2}\left(x-x_{b}(t)\right)\right) \\
& \chi_{2}(x, t)=1-\chi_{1}(x, t)
\end{aligned}
$$

In the present model there is only a single variable, the coordinate $x$ which transforms evenly under time reversal, hence, $\tilde{\chi}_{\alpha}(x, t)=\chi_{\alpha}(x, t)$. Using the eq. (49), the scalar products $\left(\chi_{\alpha}(t), L_{S}(t) \chi_{\alpha^{\prime}}(t) \rho_{0}(t)\right), \alpha, \alpha^{\prime}=1,2$ can be expressed by a single one that we denote by $q(t)$ :

$$
\begin{aligned}
\left(\chi_{1}(t), L_{S}(t) \chi_{2}(t) \rho_{0}(t)\right) & =\left(\chi_{2}(t), L_{S}(t) \chi_{1}(t) \rho_{0}(t)\right)= \\
-\left(\chi_{1}(t), L_{S}(t) \chi_{1}(t) \rho_{0}(t)\right) & =-\left(\chi_{2}(t), L_{S}(t) \chi_{2}(t) \rho_{0}(t)\right)=q(t)
\end{aligned}
$$

where $q(t)$ takes the form:

$$
q(t)=\beta^{-1} \int d x\left(\frac{\partial \chi_{1}(x, t)}{\partial x}\right)^{2} \rho_{0}(x, t)
$$

Using eq. (49) for weak noise it can further be simplified as

$$
q(t)=\frac{\omega_{b}(t) e^{-\beta V_{b}(t)}}{Z(t) \sqrt{2 \pi \beta}}
$$

The populations $n_{\alpha}(t)$ of the metastable states $\alpha=1,2$ in the frozen equilibrium distribution become at weak noise:

$$
n_{\alpha}(t)=\sqrt{\frac{2 \pi}{\beta}} \frac{e^{-V_{\alpha}(t) \beta}}{\omega_{\alpha}(t) Z(t)}
$$

Using eq. (26) this gives for the rates the instantaneous expressions [3]:

$$
\begin{aligned}
& r_{2,1}(t)=-r_{1,1}(t)=\frac{\omega_{1}(t) \omega_{b}(t)}{2 \pi} e^{-\beta \Delta V_{1}(t)} \\
& r_{1,2}(t)=-r_{2,2}(t)=\frac{\omega_{2}(t) \omega_{b}(t)}{2 \pi} e^{-\beta \Delta V_{2}(t)}
\end{aligned}
$$




\section{A. The geometric correction to the rate}

Now we come to the discussion of the corrections of the instantaneous rates that are determined by the geometric contributions $\left(\partial \chi_{\alpha}(t) / \partial t, \chi_{\alpha^{\prime}}(t) \rho_{0}(t)\right), \alpha, \alpha^{\prime}=1,2$. For the relative magnitude of the geometric correction to the instantaneous rate $r_{2,1}(t)$ one finds after some algebra:

$$
\begin{aligned}
\epsilon(t) \equiv & \frac{g_{2,1}(t)}{r_{2,1}(t)}=\frac{\left(\partial \chi_{2}(t) / \partial t, \chi_{1}(t) \rho_{0}(t)\right)}{\left(\chi_{2}(t), L_{S}(t) \chi_{1}(t) \rho_{0}(t)\right)} \\
= & -\frac{\beta}{2} \dot{x}_{b}(t) \int_{-\infty}^{\infty} d y\left(\frac{3 x_{b}(t)}{\omega_{b}^{2}(t)} y+1\right) \\
& \times \operatorname{erfc}\left(\omega_{b}(t) \sqrt{\beta / 2} y\right) \exp \left\{-\beta\left(y^{4} / 4+x_{b}(t) y^{3}\right)\right\}
\end{aligned}
$$

As expected, the error is proportional to the driving frequency $\Omega$ via the time derivative $\dot{x}_{b}(t)$. The remaining $\Omega$-dependence may be absorbed in a rescaled time $\tau=\Omega t$. An analytic solution of the integral in eq. (55) is not known. In Fig. 2 the error divided by the driving frequency is shown as it results from a numerical evaluation for different values of the driving strength $A$ and inverse temperature as a function of time. Both increasing driving strength $A$ and inverse temperature $\beta$ lead to an increase of the extrema of the error that must be compensated by a smaller driving frequency in order that the master equation with the instantaneous rates provides a valid description.

As an average measure we introduce the root mean square error $E=\left(\int_{0}^{T} d t \epsilon^{2}(t) / T\right)^{1 / 2}$ which is strictly proportional to the driving frequency $\Omega$. The proportionality factor $E / \Omega$ increases as a function of the inverse temperature first as a power law and changes to an exponential growth for large values of $\beta$, see Fig 3 .

We propose the following physical explanation of this effect: At large $\beta$ the noise is very small such that a particle that has reached at some time the top of the barrier has a very small chance to be pushed to either side by the noise. During the same time when it resides there, the potential moves such that the particle no longer will sit on top of the barrier. Depending on the direction of the motion of the potential the particle may now be on the opposite side of the barrier or on the original side where it came from. In the former case the geometric correction leads to an increase of the rate and in the latter case to a decrease of the transition rate. Because it then has again to overcome a barrier the increase of the rate is exponentially large in the inverse temperature. 

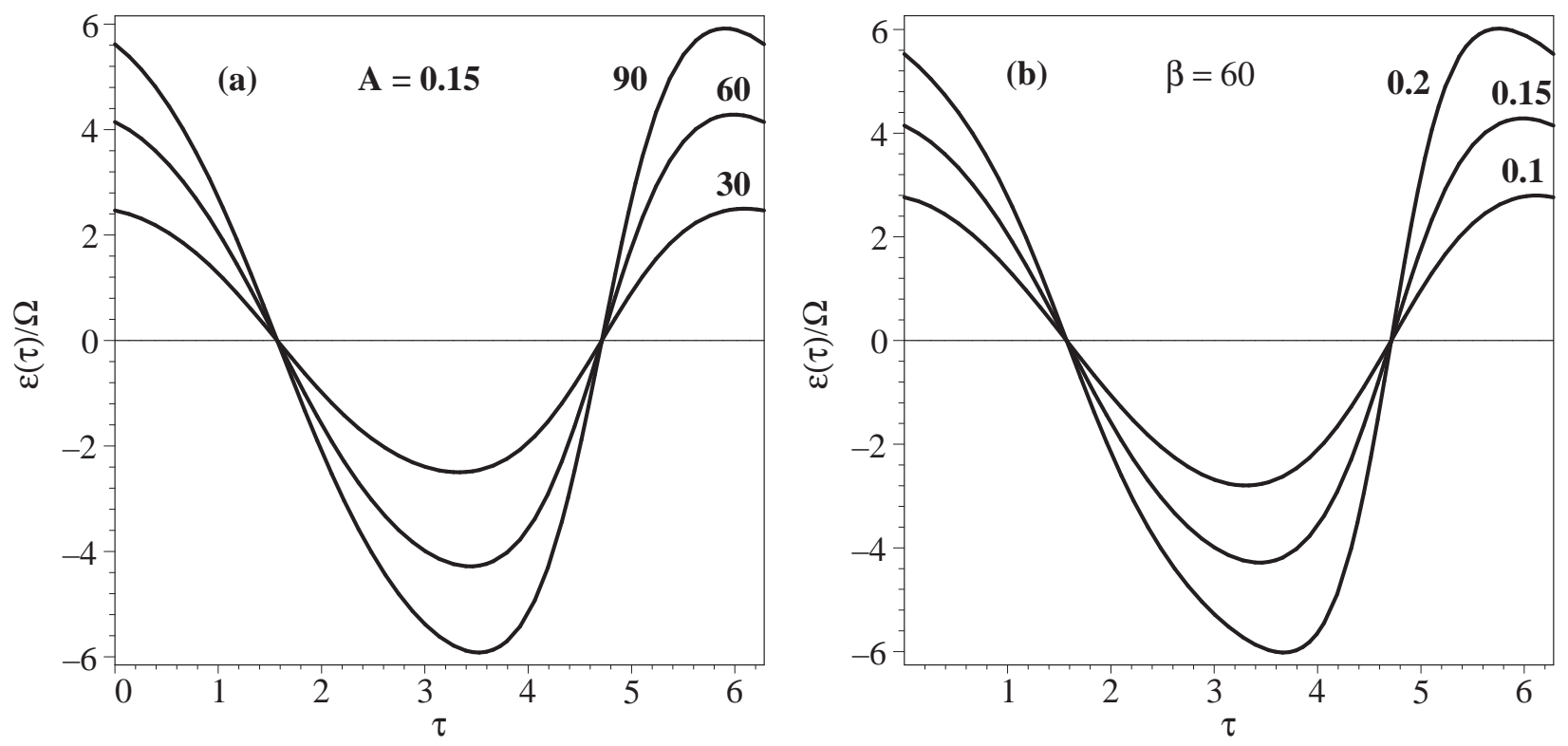

FIG. 2: The relative error of the rate oscillates as a function of time $\tau=\Omega t$. It vanishes at the extrema of the driving force. Panel (a) shows $\epsilon(\tau) / \Omega$ for different inverse temperatures $\beta=$ 30, 60, 90 at the driving strength $A=0.15$ and panel (b) for different driving strength $A=$ $0.1,0.15,0.2$ at $\beta=60$. The parameter values are indicated close to the respective curves.

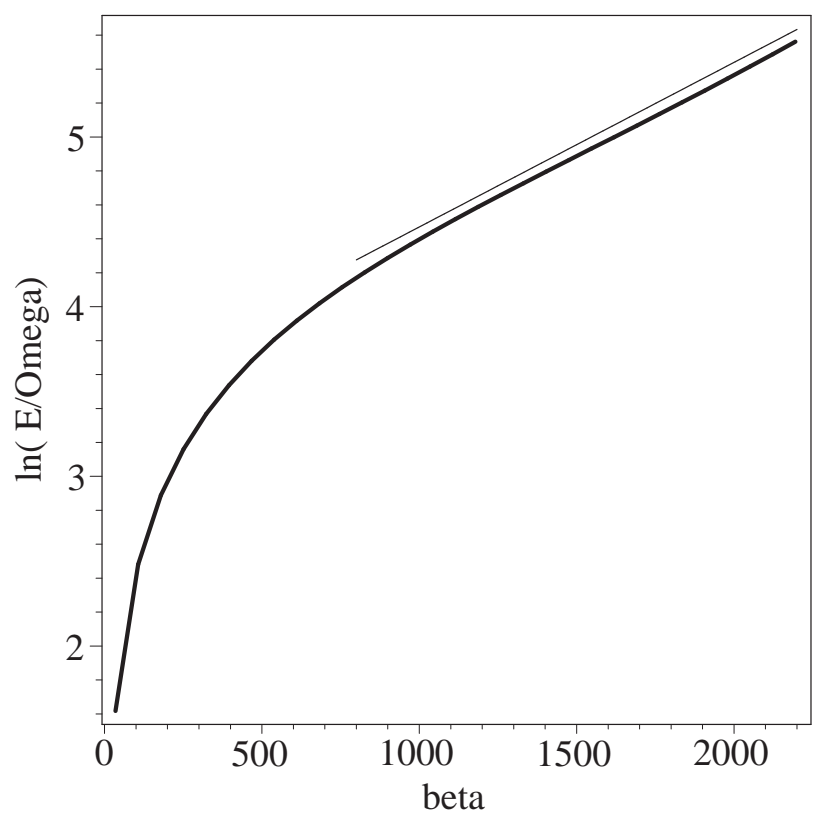

FIG. 3: The asymptotic exponential growth of the frequency independent ratio $E / \Omega$ is shown for the driving strength $A=0.12$. The straight line corresponds to a barrier height $0.97 \times 10^{-3}$. 
Finally we note that the deviation from the frozen rate on the noise strength depends on the particular model. For example in symmetric systems with varying barrier height the deviation only grows with the square root of the inverse temperature rather than exponential as in the above case where both the location and the height of the barrier changes in time.

\section{B. Average motion}

We have projected the dynamics of a a process with multiple metastable states and slowly time dependent parameters onto the slow subspace defined by the eigenfunctions of Fokker-Planck operator

As a simple example of the decoration of the two metastable states we come back to the

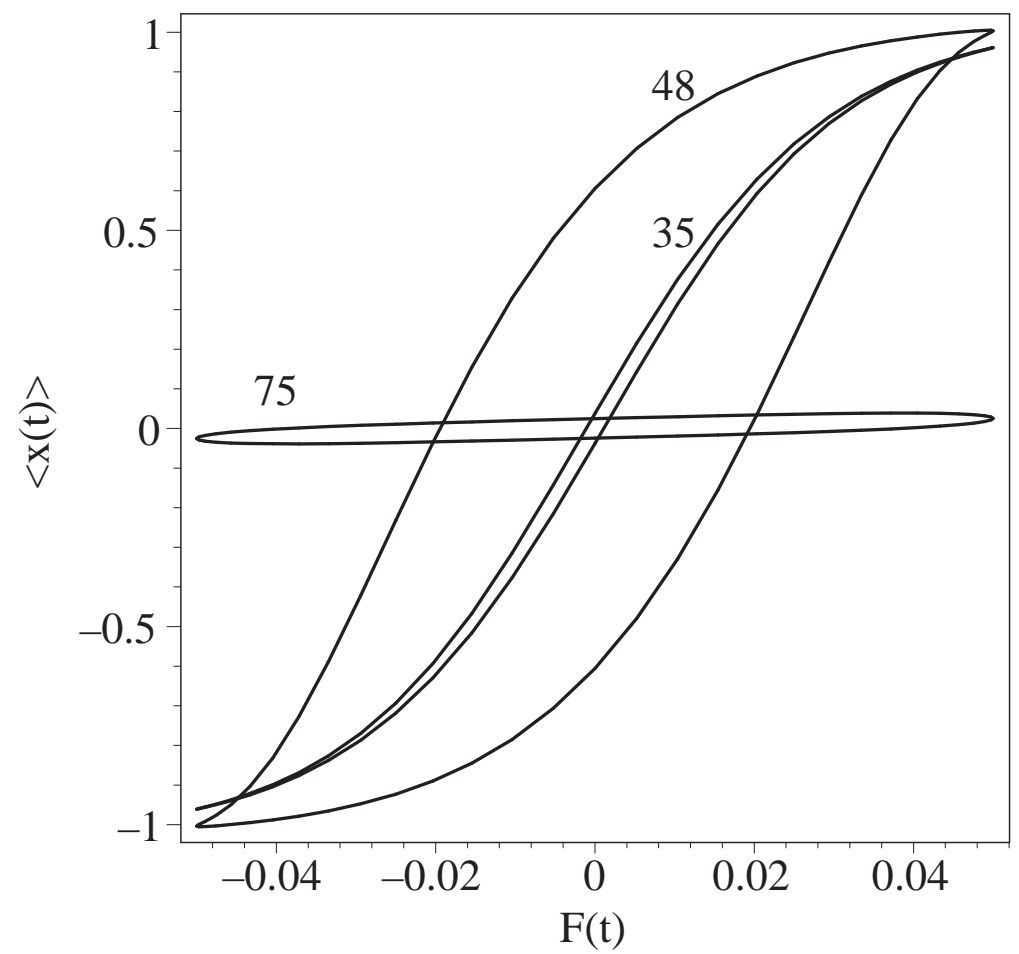

FIG. 4: The response of the average position on the driving force for the driving strength $A=0.05$ and driving frequency $\Omega=10^{-5}$ strongly depends on the magnitude of the noise. For $\beta=35$ the noise is relatively large such that the average position closely follows its adiabatic value and therefore shows almost no hysteresis; for $\beta=48$ it stays behind the force, and for $\beta=75$ it is hardly influenced by the force. The hysteresis curves are traced in the mathematically positive sense. 
bistable oscillator and consider the asymptotic motion of its average position. From eq. (32) it results as

$$
\langle x(t)\rangle=\langle x(t) \mid 1\rangle p_{1}(t)+\langle x(t) \mid 2\rangle p_{2}(t)
$$

where $\langle x(t) \mid \alpha\rangle$ denotes the conditional average of the position in the well corresponding to the metastable state $\alpha$ :

$$
\begin{aligned}
\langle x(t) \mid \alpha\rangle & =\int d x x \frac{\chi_{\alpha}(x, t)}{n_{\alpha}(t)} \rho_{0}(x, t) \\
& \approx x_{\alpha}(t)
\end{aligned}
$$

In the last equation we disregarded small contributions of the order $\mathcal{O}\left(1 /\left(\omega_{\alpha}(t) \sqrt{\beta}\right)\right)$. The probabilities $p_{\alpha}(t)$ follow as the asymptotic solutions of the master equation:

$$
\begin{aligned}
& p_{1}(t)=\frac{\int_{0}^{T} d s e^{(K(T)-K(s))} r_{1,2}(t)}{1-e^{K(T)}} e^{-K(t)}+\int_{0}^{t} d s e^{-(K(t)-K(s))} r_{1,2}(s) \\
& p_{2}(t)=1-p_{2}(t)
\end{aligned}
$$

where

$$
K(t)=\int_{0}^{t} d s\left(r_{1,2}(t)+r_{2,1}(t)\right)
$$

Rather than considering the time dependence we here show in Fig. 4 the dependence of the position on the driving force $F(t)=A \cos (\Omega t)$. In general the position lacks behind the force and therefore the dependence is hysteretic. Only if the driving is either very slow or very fast compared to the average rates there is no hysteretic behavior. In the slow case many transitions occur before a change of the potential becomes sensible. The asymptotic probabilities $p_{\alpha}(t)$ take the form of the stationary probabilities for the frozen rates. They then depend on the force but not on its rate of change. In the opposite limit the asymptotic probabilities see only the average rates and hence are time independent.

\section{SUMMARY}

In this paper we studied the dynamics of externally driven systems with multiple metastable states in the semiadiabatic limit. The full dynamics of the system comprising the fast and the slow time scales of the intrawell relaxations and of the external driving as well as of the transitions between the metastable states, respectively, is supposed to be Markovian and continuous and, hence, described by a Fokker-Planck equation. We showed 
that the long time dynamics of this Fokker-Planck equation is equivalent to the dynamics of the master equation for the transitions between the discrete metastable states. The rates that determine this master equation are time dependent taking values as if the parameters were frozen at their instantaneous values. This kind of kinetic description is not new and has long been used in the literature $[13,22]$. The present work, however, provides three new aspects. First the master equation does not result from an educated guess. It rather is obtained as the result of the projection of the dynamics onto the slow subspace of the Fokker-Planck equation. This systematic approach additionally gives a quantitative criterion when deviations from the master equation with frozen rates must be expected. This criterion does explicitly take into account the time change of the parameters and in contrast to other works [11] is not based on a comparison of time scales of the frozen dynamics with the rate of change of the parameters. As a particular result we found for the dynamics of a periodically driven overdamped Brownian oscillator that the maximal driving frequency depends on the noise strength and decreases with decreasing noise strength. Asymptotically this dependence even is exponential. Future work still has to show whether one can achieve an improvement of the dynamics if the so called geometric corrections to the rates are taken into account in cases when the time scale separation between the fast intrawell relaxation and the changes of the parameters becomes less pronounced. The third main outcome of the present approach is a partial retrieval of the underlying dynamics of the continuous variables by means of a proper decoration of the discrete states with time dependent random variables.

The necessary time scale separation forced us to exclude the occurrence of bifurcations of the deterministic dynamics caused by the change of the parameters. Such bifurcations are accompanied by a slowing down of the deterministic dynamics and at the same time by a lowering of a barrier height and consequently by a decreasing time scale of transitions. Hence, for time dependent parameters, the time scale separation between interwell and intrawell dynamics is violated within a time window around the instant of bifurcation. It seems plausible that it should be possible to cut out this time window and to bridge it by some connection condition for the probabilities of those states that are involved in the bifurcation.

Apart from the separation of the time scales, another assumption was made in the present work. First, we assumed that for fixed values of the parameters the system reaches a state of 
thermal equilibrium and hence it obeys detailed balance. We think that this assumption is not really essential for our results but it simplifies the analysis considerably. Without detailed balance more general limit sets of the dynamics can occur apart from limit points such as limit cycles and chaotic states. In general, the stationary densities are not known in absence of detailed balance, the weak noise asymptotics is plagued by notorious nonanalyticities [23], and rather little is known about transition rates in nonequilibrium systems. On the

other hand there are many important systems that are driven out of equilibrium by time dependent parameters.

\section{Acknowledgments}

The authors thank Igor Goychuk, Peter Hänggi, Sigmund Kohler, Marcin Kostur and Michael Schindler for valuable discussions and hints. This work was supported by the Deutsche Forschungsgemeinschaft (SFB438).

\section{APPENDIX A: THE CONDITIONAL PROBABILITY AT LONG TIMES}

For a sufficiently long time lag $\tau$ the conditional probability $\rho(x, t+\tau \mid y, t)$ of the continuous process $x(t)$ can be expressed in terms of the basis functions $\chi_{\alpha}(y, t)$ and $\tilde{\chi}_{\alpha}(x, t+\tau) \rho_{0}(x, t+\tau)$ spanning the slow subspaces of the backward operator at time $t$ and the forward operator at time $t+\tau$, respectively. One therefore can write:

$$
\rho(x, t+\tau \mid y, t)=\sum_{\beta, \beta^{\prime}} d\left(\beta, \beta^{\prime} ; t, \tau\right) \rho(x, t+\tau \mid \beta) \chi_{\beta^{\prime}}(y, t)
$$

where we expressed the basis function $\tilde{\chi}_{\alpha}(x, t+\tau) \rho_{0}(x, t+\tau)$ by $\rho(x, t+\tau \mid \alpha) n_{\alpha}(t+\tau)$, see eq. (34) and introduced the yet undetermined coefficients $d\left(\alpha, \alpha^{\prime} ; t, \tau\right)$ into which the time dependent factor $n_{\alpha}(t)$ is absorbed. We now multiply the conditional probability of the continuous process $\rho(x, t+\tau \mid y, t)$ with $\rho\left(y, t \mid \alpha^{\prime}\right)$, integrate over the domain of attraction $\mathcal{D}_{\alpha}(t+\tau)$ and obtain the conditional probability of the discrete process $p\left(\alpha, t+\tau \mid \alpha^{\prime}, t\right)$. Using the ansatz $(\mathrm{A} 1)$ for $\rho(x, t+\tau \mid t)$ we then find for the coefficients $d\left(\alpha, \alpha^{\prime} ; t, \tau\right)=p\left(\alpha, t+\tau \mid, \alpha^{\prime}, t\right)$. Together with eq. (A1) this yields the expression eq. (36) for the conditional probability of 
the continuous process as claimed in Sect. IV

[1] P.A.M. Dirac, Nature 192, 325 (1937); J.K. Webb et al., Phys. Rev. Lett. 87, 091301 (2001).

[2] C.M. Bender, S.A. Orszag, Advanced Mathematical Methods for Scientists and Engineers, McGraw-Hill, New York, 1978; A.H. Nayfeh, Perturbation Methods, John Wiley, New York, 1973; N. Krylov, N.N. Bogoliubov, Introduction to Nonlinear Mechanics, Princeton University Press, Princeton, 1947; H. Haken, Synergetics, An Introduction, Springer Verlag, Berlin 1983.

[3] P. Hänggi, P. Talkner, M. Borkovec, Rev. Mod. Phys. 62, 251 (1990).

[4] P. Talkner, New J. Phys. 1, 4 (1999), (http://www.njp.org/).

[5] P. Jung, Phys. Rep. 234, 175 (1993).

[6] L. Gammaitoni, P. Hänggi, P. Jung, F. Marchesoni, Rev. Mod. Phys. 70, 223 (1998).

[7] P. Reimann, Phys. Rep. 361, 57 (2002); R.D. Astumian, P. Hänggi, Phys. Today 55(11), 33 (2002).

[8] E. Evans, K. Ritchie, Biophysics J. 72, 1541 (1997); R. Merkel, Phys. Rep. 346, 343 (2001).

[9] D. Ryter, H. Meyer, Physica A 142, 122 (1987).

[10] G. Moro, J. Chem. Phys. 103, 7514 (1995).

[11] M. Bier, R.D. Astumian, Phys. Lett. A 247, 385 (1998); I. Derenyi, R.D. Astumian, Phys. Rev. Lett. 82, 2623 (1999); M. Bier, I. Derenyi, M. Kostur, R.D. Astumian, Phys. Rev. E 59, $6422(1999)$.

[12] J. Lehmann, P. Reimann, P. Hänggi, Phys. Stat. Solodi B 237, 53 (2003); J. Lehmann, P. Reimann, P. Hänggi, Phys. Rev. E 62, 6282 (2000); J. Lehmann, P. Reimann, P. Hänggi, Phys. Rev. Lett. 84, 1639 (2000).

[13] B. McNamara, K. Wiesenfeld, Phys. Rev. A 39, 4854 (1989).

[14] H. Risken, The Fokker-Planck Equation, Springer Verlag, Berlin, 1989.

[15] B.J. Matkowsky, Z. Schuss, SIAM J. Appl. Math. 42,822 (1982).

[16] P. Talkner, Z. Phys. B 68, 201 (1987).

[17] P. Talkner, Chem. Phys. 180, 199 (1994).

[18] M. Mangel, SIAM J. Appl. Math. 36, 544 (1979); D. Ryter, J. Stat. Phys. 49, 122 (1987).

[19] If there is more than one isolated saddle point on the common separatrix of the domains of attraction $\mathcal{D}_{\alpha^{\prime}}$ and $\mathcal{D}_{\alpha}$ the contribution of each saddle point to the matrixelement in eq. (15) 
can be determined separately. More complicated cases need an extra treatment, see Refs. $[3,21]$.

[20] P. Talkner, in P. Talkner, P. Hänggi (ed.), New Trends in Kramers' Reaction Rate Theory, Kluwer Academic Publishers, Dordrecht, 1995, p. 47.

[21] A.M. Berezhkovskii, P. Talkner, J. Emmerich, V.Y. Zitserman, J. Chem. Phys. 105, 10890, (1996).

[22] R.D Astumian, B. Robertson, J. Chem. Phys. 91, 4891 (1989); U. Zürcher, C.R. Doering, Phys. Rev. E 47, 3862 (1993); I.A. Goychuk, E.G. Petrov, V. May, J. Chem. Phys. 103, 4937 (1995); P. Talkner, Ann. Phys. (Leipzig), 9, 741 (2000); R. Rozenfeld, J.A. Freund, A. Neiman, L. Schimansky-Geier, Phys. Rev. E 64, 051107 (2001); R.D. Astumian, Appl. Phys. A 75, 193 (2002); P. Talkner, Physica A, in press.

[23] R. Graham, T. Tel, J. Stat. Phys. 35, 729 (1984); H.R. Jauslin, Physica A 144, 179 (1987). 